\title{
Detection of the Cardiovascular Diseases by Using a Linearly Modeling System with the PSO-based Classification Scheme
}

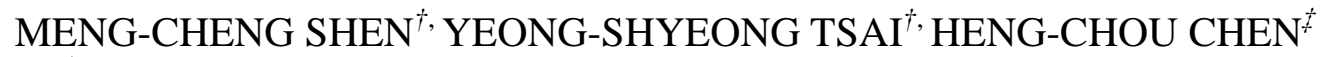 \\ ${ }^{\dagger}$ Department of Applied Mathematics, National Chung Hsing University \\ 250, Kuo Kuang Rd., Taichung 402, Taiwan, R.O.C. \\ TAIWAN \\ ${ }^{\ddagger}$ Department of Computer and Communication Engineering \\ Chienkuo Technology University \\ No. 1, Chieh Shou N. Rd., Changhua City, \\ TAIWAN
}

\begin{abstract}
In general, the detection of cardiovascular disease is performed by ECG, Electrocardiogram, to dynamically monitor and analyze the disease status. Additionally, ECG is also used to diagnose the latent disease to proceed with a further treatment. Therefore, it is very important to give a reasonable judgement from the ECG diagnosis information. In this paper, a linearly modeling system is presented to characterize both the measured ECG data and Blood Pressure Wave (BPW) information. After that, the PSO algorithm, Particle Swarm Optimization, is proposed to classify the frequency responses which are derived from the linear modeling system. From the simulation result, the successful hit rate for identifying the cardiovascular samples can reach to $80 \%$. Meanwhile, the PSO training iterations can converge under an acceptable requirement. Therefore, we can not only exactly detect the cardiovascular disease but also effectively reduce the computation time.
\end{abstract}

Key-Words :- ECG, BPW, PSO, resonance theory, linearly modeling system, LMS algorithm, adapting coefficients, zero-crossing rate of the gradient.

\section{Introduction}

The cardiovascular system is composed of the heart and blood vessels. They work together to form the blood-transporting system. Stimulated by electrocardiogram (ECG) signal, the pumping of the heart constitutes the main force of oscillation, which thus forms a blood pressure wave (BPW) propagating along the arteries.

The ECG and BPW curves are shown as in Fig. 1. By the way of using lead II process, we got the ECG signal from a test subject. At the same time, the BPW sample was collected at the twist artery of the same one. The ECG plot together with the BPW curve reveals much information related to the blood circulation information. If some blood circulation disorder occurs, it will be reflected on ECG or BPW curve. Especially for the Traditional Chinese Medicine (TCM), these two curves play a very important role in diagnosing or analyzing the diseases. Many literature papers, based on ECG and BPW information, had presented some methodologies to evaluate the healthiness of the cardiovascular system [1-2].

Wang had begun to the research on pulse wave judgement in 1977. He tried to establish the TCM pulse wave standard to unveil the physiological function of the human body. In 1987, Wang had proposed the "resonance theory" about the pulse wave. He is convinced that the pulse wave is generated by the blood pressure due to the resonant contribution among the human body organs. By using the pressure converter to obtain the pulse wave data, and adopting the Fourier Transform to analyze them, he discovered that there exists a strong relationship between the resonance intensity and the healthiness of the human body organs.

From the theory of "The Resonance Effect on Blood Pressure and Flow Hypothesis" presented by W.K. Wang and Y. Y. Lin, we know there are resonant frequencies in vitals. When the blood flows into organ along with pulse vibration, blood plexus not only has resonance phenomenon, but also initiates organ's mechanical micro-vibration by itself. Each organ has its special principal resonant frequency; the circulatory system is harmonious and stable. When an organ was excited, the resonance frequency changes [3-5].

In discussion about the resonance variation and 
physiological reaction, it was discovered that a patient with cardiovascular disease will bring some disorder signals on ECG and BPW curves. It is like the AM and FM noises which are acting on the five major resonance frequencies [6]. In addition to the observation of the five major frequencies, we are convinced that there still exists some information not having been discussed inside the ECG or BPW. In this paper, we attempted to represent the cardiovascular system by a linearly modeling system. The input signal of linearly modeling system is ECG, the output signal BPW. The ECG and BPW signal samples are obtained prior to the overall simulation. And then, the frequency response is computed based these signal samples. The eigenvalues of the frequency response are extracted. Finally, we try to compare the difference of the eigenvalues between the healthy samples and the patient ones. Also, we identified the healthy subjects and cardiovascular patients by using the PSO-based scheme to decrease the clustering computation time.
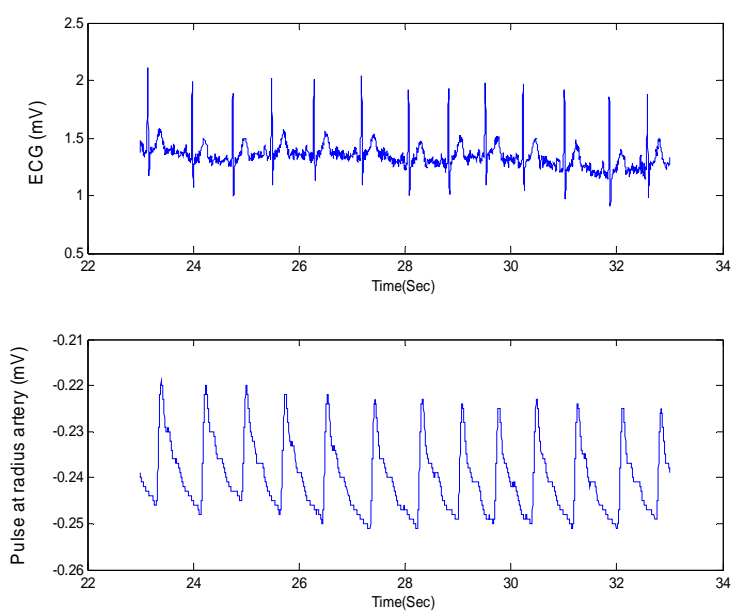

Fig. 1 The ECG and blood pressure wave (BPW) are shown.

\section{Linearly modeling system and PSO algorithm}

In this paper, we analyze the complex blood circulation system from the macroscopic system viewpoint to search for a sign of disease. The linearly modeling system is applied to represent the frequency response between ECG. and BPW. Then, by coherence analysis, we study its coherence power transfer, and further extract the characteristic parameters of the system. Another main scheme, particle swarm optimization (PSO), is used in the classification process to separate the healthy subjects from the abnormal ones.
The blood circulation is represented by a linearly modeling system whish is actually an adaptive digital filter.

Generally, adaptive digital filters are used in cases requiring system identification, such as acoustic echo cancellation, active noise control and communication channel equalization [7]. The adaptive filters need real-time responses in these applications. LMS and Recursive Least Square (RLS) algorithms are the two most commonly-used adaptive algorithms [8]. LMS has a low computation complexity which is proportional to the number of taps $\mathrm{M}$ in the filter, but its convergence rate slows down due to input signal correlation. RLS has a fast convergence rate but requires an enormous computation power proportional to $M^{2}$. Notably, in acoustic echo cancellation under an $8 \mathrm{kHz}$ sampling rate, the adaptive filter would require up to around 2,000 coefficients to estimate adequately the spatial impulse response of a room. Hence, the RLS algorithm may use up the hardware's computation resources to estimate the response. On the other hand, while LMS has a slower convergence rate than RLS, its low computation complexity still makes it a popular algorithm. Many studies have attempted to enhance the convergence performance of the LMS algorithm by either raising its convergence rate or lowering its estimation error.

Fig. 2 depicts a block diagram of system identification using adaptive filtering. The LMS algorithm includes three processes: filtering output, computing error and adapting coefficients [9]. Hence, the duration for sampling an input signal and generating an output signal can be divided into three stages as described below:

Filtering output

$y(n)=\mathbf{w}^{T}(n) \mathbf{x}(n)$,

Computing error

$e(n)=d(n)-y(n)$,

Adapting coefficients

$\mathbf{w}(n+1)=\mathbf{w}(n)+\mu e(n) \mathbf{x}(n)$

The term $\mathbf{x}(n)=[x(n), x(n-1), \cdots, x(n-M+1)]$ denotes the input vector; $d(n)$ represents the actual output of an unknown system $\mathbf{w}(n)$, and $y(n)$ is the output of the filter employed to estimate $d(n)$. Additionally, $e(n)$ given by the difference between $d(n)$ and $y(n)$, represents the filter's estimation error, and $\mu$ denotes the step size controlling the adaptation amount of filter coefficients.

\subsection{Linearly modeling system}




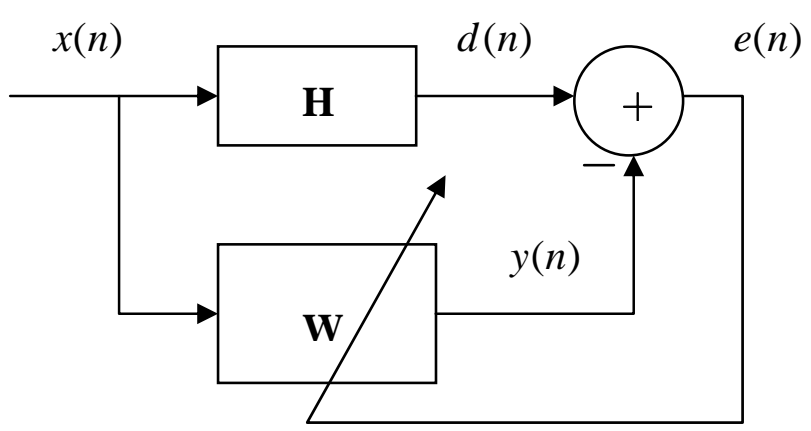

Fig. 2 System identification block diagram.

By way of the adaptive digital filter, we can understand the system impulse response. The system characteristic is totally reflected on the impulse response. Therefore, the analysis of an unknown system can be completed by an impulse response especially for system identification issues. Besides, the system transfer function and frequency response are derived from impulse response. In this paper, we will refer to the adaptive digital filter as a linearly modeling system. The input is ECG similarly as $x(n)$ shown in Fig. 2, the output BPW similar to $y(n)$ expressed in Eq. (1). It is like the BPW which is induced by ECG.

\subsection{PSO algorithm}

Particle swarm optimization (PSO), first introduced by Kennedy and Eberhart [10] in 1995, is a recent addition to the list of global search methods. It is a population-based stochastic optimization technique with self-adaptive mechanism based on the movement of swarms and inspired by social behavior of bird flocking or fish schooling. In the past several years, PSO has been successfully applied in many different application areas, such as design of antennas of communication systems, system identifications on control engineering, etc, due to its robustness and simplicity [11]. Instead of using genetic operations, such as crossover and mutation of genetic algorithms (GA), to manipulate the individuals, each particle in PSO flies in the search space with velocity which is dynamically adjusted according to its own flying experience and its flying companion's experience. As a result, trajectory of each individual is influenced by its own successful experiences, as well as the successes of its neighbors. Toward the end of the optimization, most particles converge to the global optimum, which expectedly results into the best design. In comparison to GA, PSO has a simpler configuration with fewer complicated operations during the evolution process. Because of these advantages, PSO has received increasing attention in recent years.
The PSO is initiated by randomly allocating particle positions in the solution space. Assume that there are $\mathrm{I}$ particles in a swarm $\mathbf{X}(t)=\left\{\begin{array}{llll}\mathbf{x}_{1}(t) & \mathbf{x}_{2}(t) & \cdots & \left.\mathbf{x}_{I}(t)\right\}\end{array}\right.$ at instant t, where $\mathbf{x}_{i}(t)$ represents the $i^{t h}$ particle in the search space. At each iteration, the particles' velocities are calculated according to

$$
\begin{aligned}
\mathbf{v}_{i}(t+\Delta t)=w \cdot \mathbf{v}_{i}(t) & +c_{1} \cdot \operatorname{rand}() \cdot\left[\mathbf{x}_{i}^{\text {best }}(t)-\mathbf{x}_{i}(t)\right] \\
& +c_{2} \cdot \operatorname{rand}() \cdot\left[\mathbf{x}^{\text {best }}(t)-\mathbf{x}_{i}(t)\right],
\end{aligned}
$$

and the particles' positions are updated by

$\mathbf{x}_{i}(t+\Delta t)=\mathbf{x}_{i}(t)+\mathbf{v}_{i}(t+\Delta t) \cdot \Delta t$

where $\Delta t$ is the duration between iterations, $w$ represents an inertia coefficient, $c_{1}$ and $C_{2}$ are acceleration constants, $\operatorname{rand}()$ is a function generating random numbers with uniform distribution from within $\left[\begin{array}{ll}0 & 1\end{array}\right], \quad \mathbf{x}_{i}^{\text {best }}(t)$ and $\mathbf{x}^{\text {best }}(t)$ represent the best ever position of particle i (i.e. local best) and the global best position in the swarm at instant $\mathrm{t}$, respectively. For problems with $\mathrm{N}$ parameters to be optimized, each particle comprises $\mathrm{N}$ factors, i.e., $\quad \mathbf{x}_{i}=\left(\begin{array}{llll}x_{i, 1} & x_{i, 2} & \cdots & x_{i, N}\end{array}\right)$. Velocities associated with $\mathbf{x}_{i}$ are accordingly represented as $\mathbf{v}_{i}=\left(\begin{array}{llll}v_{i, 1} & v_{i, 2} & \cdots & v_{i, N}\end{array}\right)$.

As shown in Eqs. (4) and (5), there are 3 terms affecting the velocities of each particle in a swarm, in which the first term is relevant to the velocities in the previous generation, while the second and the third terms are closely related to the best ever position of particle $\mathrm{i}$ and global best position in the swarm, respectively. Based on the evolutionary mechanism, particles are evolved toward the attainment of global optimal solutions with the help of the 'local best' of each particle and 'global best' of the swarm, taking account the spontaneity of particles as well as the evolutionary trend of the swarm.

Particles in a conventional PSO evolve according to the velocity-updating rule in Eq. (4), which takes account of the local best $\mathbf{x}_{i}^{\text {best }}(t)$ and global best $\mathbf{x}^{\text {best }}(t)$ without reference to the evolution experience of other particles in the swarm. It suffices to say that each particle evolves independently. To improve the particle's velocity to efficiently evolve to the optimal positions, conventional approaches generally achieve this objective by increasing the number of particles in a swarm. However, the increase of particle numbers inevitably demands extra computation in each iteration, resulting in longer evolution time required 
to obtain the results. For objective functions with more complexities, the situation will be even worse. As attempts to solve this problem, several techniques were proposed by altering the inertia coefficient [11] and acceleration constants [12] to improve the velocities during evolution process. Simulation results, however, show inconsistent evolution performances for different objective functions by using these techniques.

\section{Computer Simulations}

The ECG and BPW signal samples are extracted by PowerLab Systems of the ADInstruments Corp.

The ECG of lead II and BPW of a radial artery non-invasively detected at the wrist using a piezoelectric transducer were simultaneously recorded for 10s and digitized at a sampling rate of $1000 \mathrm{~Hz}$. The $3 \mathrm{~dB}$ frequencies of the low-pass filter are $50 \mathrm{~Hz}$ for ECG and BPW.

The study subjects were composed of two groups. The first group consisted of 20 healthy subjects whose health checks are normal and without any reported cardiovascular disease. The second group was composed of 20 patients with cardiovascular-related diseases. All the subjects were rested for about twenty minutes to get a steady pulse waveform, and then detected their ECG and BPW signals by the instruments descried above.

Each test subject can have a pair of output signals, ECG and BPW. After further computation of these two signals by linearly modeling system, we can then obtain the adapting coefficients as in (3). The adapting coefficients are shown in Fig. 3. Since it is not obvious to compare the characteristic difference in time domain among these adapting coefficients, the adapting coefficients are converted into frequency domain which governs signal characteristic by amplitude and frequency. In Fig. 4, the amplitude and frequency of each adapting coefficient are demonstrated.

It is worth noting that there exists zero-crossing rate of the gradient for the amplitude and phase curves. In the following, an evolutionary technique PSO is adopted to cluster the zero-crossing rate of gradient for the corresponding healthy samples and patient ones.

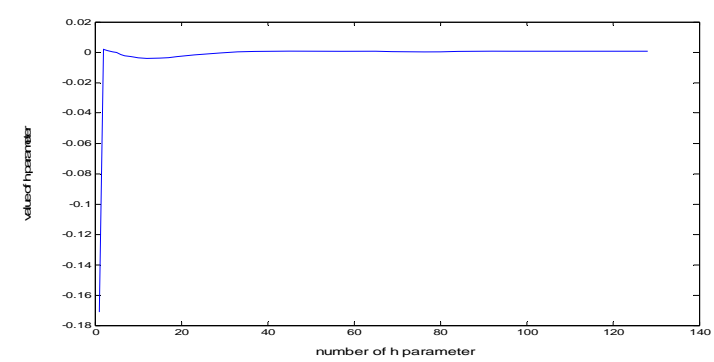

Fig. 3 Adapting coefficients of a linearly modeling system
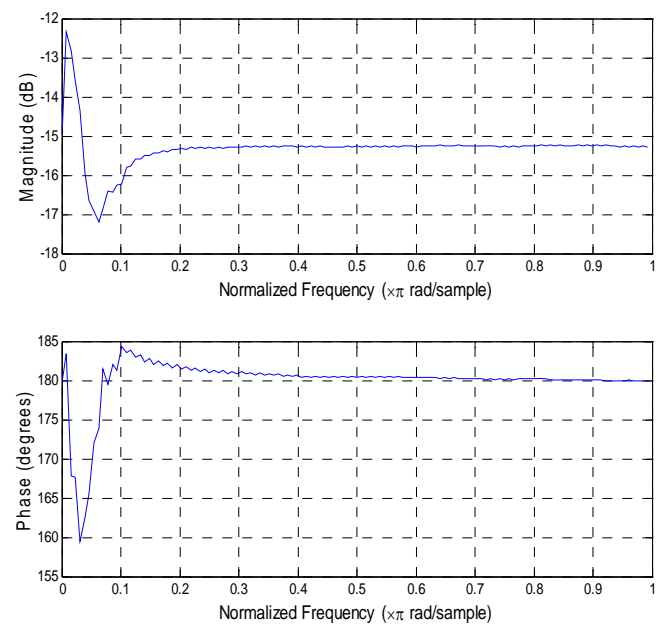

Fig. 4 The frequency responses of the adapting coefficients

The clustering between healthy samples and patient ones is performed as follows.

Step 1 Each pair of the ECG and BPW signals from one individual are fed into the linearly modeling system. The BPW is the output of the linearly modeling system. By using least mean square algorithm, we then calculate the adapting coefficients, $h_{1} h_{2} \ldots h_{n}$, where $n=128$.

Step 2 Converting every adapting coefficients, $h_{1} h_{2} \ldots h_{n}$ into the signal amplitude and phase frequency response.

Step 3 Count the zero-crossing rate along the curve of both signal amplitude and phase, and let it be the eigenvalues.

Step 4 According to the zero-crossing rate in both of signal Phase and Amplitude, we calculate the mean value $u_{1}$ for both the healthy samples and $u_{2}$ for the patient ones, respectively.

Step 5 By using PSO algorithm, a point $u_{p}$ on the line connecting $u_{1}$ and $u_{2}$ can be obtained. Try to find the most suitable slope of this line passing the $u_{p}$. After PSO optimization process, an optimal separating line which passes $u_{p}$ can be found.

Since the previous coefficients of the phase and amplitude curves have much more variation, we focus on zero-crossing rate only along with the previous 30 coefficients. In Fig. 5, the 
horizontal-axis stands for the zero-crossing rate of phase, and vertical-axis the zero-crossing rate of amplitude. Besides, symbol ' $\circ$ ' denotes the healthy samples and ' $x$ ' the patient ones. Additionally, there may not involve whole ' $\circ$ ' or ' $x$ ' notations as the original test samples because some test samples with the same characteristic values will locate on identical positions.

In Fig. 5, the mean values of $u_{1}$ and $u_{2}$ are connected by solid line $L_{1}$. The dotted line $L_{2}$ represents a separating cut to distinguish the ' $\circ$ ' and ' $x$ ' samples. We can find that PSO algorithm can be successfully applied on the identification of cardiovascular system disease. The final identification rate can reach to $80 \%$. Meanwhile, only about 10 iterations are required for the overall simulation to find the optimal point $u_{p}$.

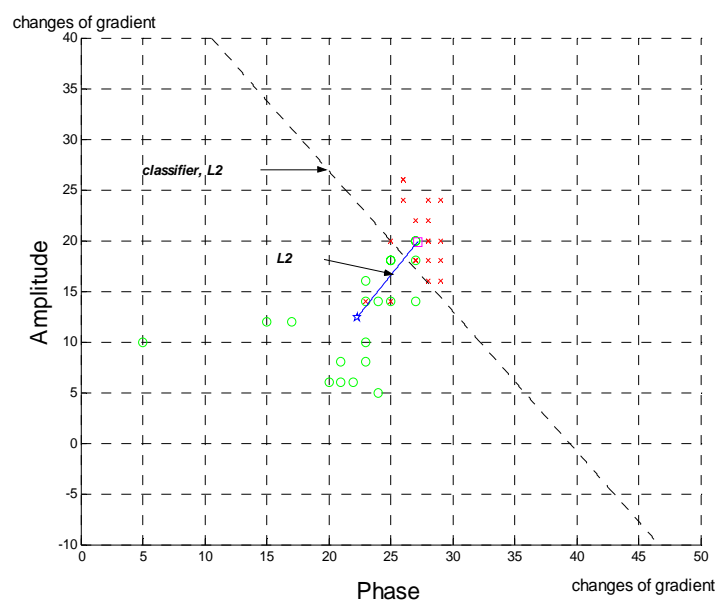

Fig. 5 The identification results by using the proposed scheme. The ' $\circ$ ' notations represent health samples and ' $x$ ' represent abnormal samples.

\section{Conclusions}

Since the heart and arterial system play an important role in the human body, cardiovascular-related diseases have been one of the major causes of death. Furthermore, they often cause sudden death without warning. To identify the initial pathological sign of a disease in advance, it is helpful to study the system characteristics. Regarding the system level analysis, it is particularly important to comprehend the power flow from input to output.

In this paper, the cardiovascular system is simulated by the linearly modeling system. Based on the modeling system, the cardiovascular disease samples can be successfully identified. We discovered that either signal amplitude or phase of the frequency response of the healthy samples is significantly smaller than that of the cardiovascular disease samples. In order to effectively classify those samples with the eigenvalues, the mean value $u_{1}$ for both the healthy samples and $u_{2}$ for the patient ones were calculated respectively. After that, we connected $u_{1}$ and $u_{2}$, and applied the PSO on the line to search a optimal position of classification. Through the PSO clustering, the identification rate can reach to $80 \%$. We had also tried the k-means approach to cluster the zero-crossing rate of gradient sample. Nevertheless, due to the position mixing of the zero-crossing rate for both the healthy samples and the patient ones, k-means algorithm can not present a better identification rate.

In summary, the results provide us with a new scope to study blood circulation using the linearly modeling system. It should be noted that for each human body organ, there may exist difference in frequency response derived from ECG or BPW. We also observed that the zero-crossing rate of the corresponding frequency response should be used as a measured index. If we can establish the database of the zero-crossing rate of gradient, it will become very useful and convenient to discriminate healthy cases from patients in detecting the cardiovascular-related diseases.

\section{References:}

[1] Albert Chin-Yuh Lin, "The Development of Data Acquisition System for Chinese Medical Pulse Diagnosis," Traditional Chinese Medicine Annual Report, Taiwan, R.O.C., 1998, pp. 307-346.

[2] J. J. Chen, "Application of the Principle Pulse Diagnostics in Chinese Medicine and Measured Pulse Wave Signals to Clinical Diagnoses," Traditional Chinese Medicine Annual Report, Taiwan, R.O.C., Vol.3, No. 24, 2006.

[3] W. K. Wang, Y. Y. Lo, T. L. Hsu and Y. Y. Lin Wang, "Resonance of Organs with the Heart," Biomedical Engineering An International Symposium, Hemisphere Publish, New York, 1989, pp. 259.

[4] Y. Y. Lin Wang, M. Y. Jan, C. S. Shyu, C. A. Chiang and W. K. Wang, "The natural frequencies of the arterial system and their relation to the heart rate," IEEE Trans. Biomed. Eng., Vol.51, 2004, pp. 193-195.

[5] Y. Y. Lin, C.C. Chang, J.C. Chen, H. Hsiu and W.K. Wang, "Pressure Wave Propagation in Arteries- A Model with Radial Dilatation for Simulating the Behavior of a Real Artery," IEEE Engineering in Medicine and Biology., Vol.16, 1997, pp. 51-56.

[6] C. C. Wei, Pei-Chen Lo and Lin-Kun Wu, 
"System Analysis of Blood Circulation Based on Coherence Power Transfer: From Electrical Driving to Mechanical Vibration," Japanese Journal of Applied Physics, Vol. 45 No. 4A, 2006, pp.2863-2868.

[7] H. Hahn, B.J . Hosticka and D. Timmermann, "Alternative signal processor arithmetic for modified implementation of a normalized adaptive channel equalizer," IEE Proceedings F - Radar and Signal Processing, Vol. 139, No. 1, Feb. 1992, pp. $36-42$.

[8] Heng-Chou Chen and Oscal T.-C. Chen, "A predictive updating scheme to improve the NLMS algorithm for Acoustic echo cancellation," Proc. of IEEE International Symposium on Circuits and Systems, Vol. 3, 1999, pp. 552-555.
[9] S. Haykin, Adaptive Filter Theory, New York: Prentice-Hall, 4th ed., 2002.

[10] J. Kennedy and R. Eberhart, "Particle swarm optimization," Proc. IEEE Int. Conf. Neural Networks, 1995, pp. 1942-1948.

[11] W.-P. Huang, L.-F. Zhou and J.-X. Qian, "FIR filter design: frequency sampling filters by particle swarm optimization algorithm," Proceedings of the IEEE Third International Conference on Machine Learning and Cybernetics, Aug. 2004, pp. 2322-2327.

[12] D.J. Krusienski and W. K. Jenkins, "Design and performance of adaptive systems based on structured stochastic optimization strategies," IEEE Circuits and Systems Magazine, First Quar ter2005, pp. 8-20. 